Lance A. Fisher ${ }^{1}$ / Hyeon-seung Huh ${ }^{2}$

\title{
Combining sign and parametric restrictions in SVARs by utilising Givens rotations
}

\author{
${ }^{1}$ Department of Economics, Macquarie University, Sydney 2109, Australia, E-mail: lance.fisher@mq.edu.au \\ ${ }^{2}$ School of Economics, Yonsei University, 50 Yonsei-ro, Seodaemun-gu, Seoul 03722, Republic of Korea, Phone: +82-2-2123- \\ 5499, E-mail: hshuh@yonsei.ac.kr
}

\begin{abstract}
:
This paper shows how to impose parametric restrictions in conjunction with sign restrictions to separate the shocks in SVARs. In sign restrictions, it is common to rotate an initial set of orthogonal shocks by utilising a Givens rotation matrix. In this paper, we show how to construct the Givens rotation matrix when parametric restrictions are part of the identification in sign restricted SVARs. The properties of Givens matrices are such that the parametric restrictions imply a system of equations which can be solved for the unknown parameters (or "angles") in a rotation matrix, conditional on the values of the parameters which are drawn. The Givens rotation matrix formed in this manner is such that the parametric restrictions on the impulse responses are satisfied on each draw in sign restrictions. The method is demonstrated in an influential SVAR and is shown to generate results similar to those from a recent method which imposes the orthogonality and zero parametric restrictions on the columns of the rotation matrix in sign restrictions.
\end{abstract}

Keywords: Givens rotations, QR decomposition, sign and parametric restrictions, structural vector autoregressions

JEL classification: C32, C51, E32

DOI: 10.1515/snde-2018-0104

\section{Introduction}

This paper develops a method to separate the shocks in SVARs which combines parametric and sign restrictions on the impulse responses. The shocks in SVARs have traditionally been identified by parametric restrictions. These can take the form of exclusion restrictions on the contemporaneous and/or long-run impacts of the shocks on some of the variables. Contemporaneous zero restrictions are often justified on the grounds that some variables are sluggish to adjust to new information or that new information is observed with a delay. Long-run zero restrictions are typically justified on the grounds of long-run neutrality propositions in economic theory. Recently, the sign restriction methodology for separating the shocks in SVARs has become popular. The sign restrictions approach was first introduced by Faust (1998), Canova and De Nicoló (2002), and Uhlig (2005).

The sign restrictions methodology involves rotating an initial set of orthogonal shocks in a SVAR to form a new set of orthogonal shocks from which impulse responses are obtained. These are retained if the impulse responses accord with the sign restrictions and discarded if they do not. The procedure is repeated by taking another rotation of the initial orthogonal shocks from which another set of impulse responses are obtained and judged by the sign restrictions. The rotation of the initial orthogonal shocks in sign restrictions is often accomplished by utilising a Givens rotation matrix. A contribution of the paper is that we construct the Givens rotation matrix for the case of full sign identification so that it is analogous to the rotation matrix from a QR decomposition in which case both rotations would be expected to give very similar results. This may not necessarily be the case for Givens matrices constructed somewhat differently, for example, as in Peersman (2005).

The primary objective of this paper is to show how to combine parametric restrictions with sign restrictions when the rotation matrix is of a Givens form. Baumeister and Benati (2013) impose a single zero contemporaneous restriction in a sign restrictions framework that utilises a Givens rotation matrix and their approach is extended by Haberis and Sokol (2014) to multiple zero contemporaneous restrictions. In this paper, we fully develop the method for combining parametric restrictions with sign restrictions utilising Givens matrices. The parametric restrictions can be long-run and/or contemporaneous restrictions. The method has the feature that for a SVAR of $n$ variables, at most $n-1$ parametric restrictions can be placed on the first shock, $n-2$ on the second shock and so forth. The method is developed in the context of Peersman's (2005) four variable SVAR for the 
United States (US), although it can be applied to a SVAR of any number of variables. We introduce four longrun neutrality restrictions into Peersman's sign identified SVAR to develop the method. A contemporaneous zero restriction is then added to this identification to further demonstrate the procedure. The responses from Peersman's sign identified SVAR with the four long-run zero restrictions are quite reasonable but when the contemporaneous restriction is added, puzzles in the responses emerge.

The method is tractable because of the properties of Givens matrices. These properties are such that the parametric restrictions imply a system of equations which can be solved for the unknown parameters or "angles" in the Givens matrices, conditional on the values of the parameters which are generated in sign restrictions. The Givens rotation matrix formed in this manner is such that the parametric restrictions on the impulse responses are satisfied on each draw in sign restrictions. We relate this method to the recent procedure of Arias, RubioRamírez, and Waggoner (2018), hereafter ARW, for combining zero parametric restrictions with sign restrictions. They impose the zero parametric restrictions, along with the orthogonality restrictions, on the columns of the rotation matrix whereas in the Givens method, the rotation matrix is already orthogonal by the properties of Givens matrices. We apply their method to the SVAR with signs and the four long-run zero restrictions and find that the responses are similar to those from our Givens rotation method.

The structure of the paper is as follows. Section 2 presents the SVAR. Section 3 discusses Givens matrices and their properties. Section 4 shows the method to obtain the Givens rotation matrix in the full sign identification case in Peersman's SVAR. Section 5 develops the method to combine sign with parametric restrictions using Givens matrices in the SVAR with signs and four long-run neutrality restrictions. Section 6 demonstrates the method when the identification is extended to include a contemporaneous zero restriction. Section 7 presents the empirical results. Section 8 describes the Arias, Rubio-Ramírez, and Waggoner (2018) method in the SVAR with signs and the four long-run neutrality restrictions and presents the results. Section 9 compares the two methods and discusses their relative applicability and computational efficiency. Section 10 provides a summary.

\section{The structural model}

The methods in this paper are applied to the four-variable vector-autoregressive (VAR) model of Peersman (2005). In Peersman's model, the variables are the price of oil $\left(o_{t}\right)$, output $\left(y_{t}\right)$, and consumer prices $\left(p_{t}\right)$, in natural logs, and the short-term interest rate $\left(i_{t}\right)$. Peersman treats the oil price, output, and consumer prices as $\mathrm{I}(1)$ variables and the interest rate as an $\mathrm{I}(0)$ variable. The $\mathrm{I}(1)$ variables are differenced once before entering the VAR so that the vector of variables in the VAR is $z_{t}=\left(\begin{array}{lllll}\Delta o_{t} & \Delta y_{t} & \Delta p_{t} & i_{t}\end{array}\right)^{\prime}$. The reduced-form vector moving average representation is:

$$
z_{t}=D(L) e_{t}
$$

where $D(L)=\left(I+D_{1} L+D_{2} L^{2}+\ldots\right), L$ is the lag operator, and $e_{t}$ is distributed as $N(0, \Omega)$ so that the reduced-form errors have a mean of zero and are contemporaneously correlated. An initial set of orthogonal shocks is formed as $\varepsilon_{t}=A_{0} e_{t}$ where the matrix $A_{0}^{-1}$ is such that $\Omega=A_{0}^{-1}\left(A_{0}^{-1}\right)^{\prime}$. In this paper, the matrix $A_{0}^{-1}$ is obtained from an eigenvalue decomposition of $\Omega$, but it could also be obtained from a Cholesky decomposition of $\Omega$, in which case it is lower triangular. Write equation (1) as:

$$
z_{t}=D(L) A_{0}^{-1} A_{0} e_{t}
$$

The initial orthogonal shocks are rotated using the Givens rotation matrix $G$, which has the property that $G G^{\prime}$ $=G^{\prime} G=I$, to form a new set of orthogonal shocks. The matrix $G$ is $(4 \times 4)$ as the model has four variables. Now write equation (2) as:

$$
z_{t}=D(L) A_{0}^{-1} G G^{\prime} A_{0} e_{t}
$$

The new set of orthogonal shocks is $\varepsilon_{t}=G^{\prime} A_{0} e_{t}$, and the impulse responses to them are given by:

$$
C(L)=D(L) A_{0}^{-1} G
$$

The long-run response of the variables to the new orthogonal shocks is:

$$
C(1)=D(1) A_{0}^{-1} G
$$


and the contemporaneous response is:

$$
C(0)=A_{0}^{-1} G
$$

since $D(0)=I$. We now turn to the properties of Givens matrices.

\section{Properties of Givens matrices}

In the four variable case, there are six Givens matrices. They are $G\left(\theta_{12}\right), G\left(\theta_{13}\right), G\left(\theta_{14}\right), G\left(\theta_{23}\right), G\left(\theta_{24}\right)$ and $G\left(\theta_{34}\right)$. They are formed by taking the $(4 \times 4)$ identity matrix and setting $G^{i i}\left(\theta_{i j}\right)=\cos \theta_{i j}, G^{i j}\left(\theta_{i j}\right)=-\sin \theta_{i j}, G^{j i}\left(\theta_{i j}\right)=\sin$ $\theta_{i j}, G^{j j}\left(\theta_{i j}\right)=\cos \theta_{i j}$, where the superscripts refer to the row and column of $G\left(\theta_{i j}\right)$. For example, the Givens matrix $G\left(\theta_{34}\right)$ is

$$
G\left(\theta_{34}\right)=\left[\begin{array}{cccc}
1 & 0 & 0 & 0 \\
0 & 1 & 0 & 0 \\
0 & 0 & \cos \theta_{34} & -\sin \theta_{34} \\
0 & 0 & \sin \theta_{34} & \cos \theta_{34}
\end{array}\right]
$$

To be economical in notation, we write $c_{i j}$ for $\cos \theta_{i j}$ and $s_{i j}$ for $\sin \theta_{i j}$ so, for example, $G\left(\theta_{34}\right)$ is written as:

$$
G\left(\theta_{34}\right)=\left[\begin{array}{cccc}
1 & 0 & 0 & 0 \\
0 & 1 & 0 & 0 \\
0 & 0 & c_{34} & -s_{34} \\
0 & 0 & s_{34} & c_{34}
\end{array}\right]
$$

The Given matrices have the property that they are orthogonal so that $G\left(\theta_{i j}\right)^{\prime} G\left(\theta_{i j}\right)=G\left(\theta_{i j}\right) G\left(\theta_{i j}\right)^{\prime}=I$. The Givens rotation matrix $G$ that is used to rotate the initial set of orthogonal shocks $\left(A_{0} e_{t}\right)$ to form the new set of orthogonal shocks $\left(G^{\prime} A_{0} e_{t}\right)$ is constructed as the product of the six Givens matrices, i.e. as:

$$
G=\left[G\left(\theta_{12}\right) G\left(\theta_{13}\right) G\left(\theta_{14}\right)\right]\left[G\left(\theta_{23}\right) G\left(\theta_{24}\right)\right]\left[G\left(\theta_{34}\right)\right]
$$

and it has the property that $G^{\prime} G=G G^{\prime}=I$.

The matrix $G$ has the form:

$$
G=\left[\begin{array}{cccc}
c_{12} c_{13} c_{14} & * & * & * \\
s_{12} c_{13} c_{14} & * & * & * \\
s_{13} c_{14} & * & * & * \\
s_{14} & * & * & *
\end{array}\right]
$$

which shows that the first column of $G$ depends only on the angles $\theta_{12}, \theta_{13}$ and $\theta_{14}$. We will also consider the matrix $\bar{G}$ defined as:

$$
\bar{G}=\left[G\left(\theta_{23}\right) G\left(\theta_{24}\right)\right]\left[G\left(\theta_{34}\right)\right]
$$

It has the form:

$$
\bar{G}=\left[\begin{array}{cccc}
1 & 0 & 0 & 0 \\
0 & c_{23} c_{24} & * & * \\
0 & s_{23} c_{24} & * & * \\
0 & s_{24} & * & *
\end{array}\right]
$$

The first column is the unit basis vector and the second column depends only on the angles $\theta_{23}$ and $\theta_{24}$. We note from equation (8) that the first two columns of $G\left(\theta_{34}\right)$ are unit basis vectors and the third column depends only on the angle $\theta_{34}$.

We will utilise the following two results. 


\section{Result 1}

The first column of the matrix $G$ is identical to the first column of the matrix $G^{1}$ where $G^{1}=$ $G\left(\theta_{12}\right) G\left(\theta_{13}\right) G\left(\theta_{14}\right)$.

\section{Proof}

Since $G=G^{1} \bar{G}$ and the first column of $\bar{G}$ is the unit vector $\left(\begin{array}{llll}1 & 0 & 0 & 0\end{array}\right)^{\prime}$, the first column of $G^{1}$ becomes the first column of $G$.

\section{Result 2}

The first two columns of the matrix $\bar{G}$ are identical to the first two columns of the matrix $G^{2}$ where $G^{2}=$ $G\left(\theta_{23}\right) G\left(\theta_{24}\right)$.

\section{Proof}

Since $\bar{G}=G^{2} G\left(\theta_{34}\right)$ and the first two columns of $G\left(\theta_{34}\right)$ are $\left(\begin{array}{lllll}1 & 0 & 0 & 0\end{array}\right)^{\prime}$ and $\left(\begin{array}{lllll}0 & 1 & 0 & 0\end{array}\right)^{\prime}$, respectively, the first and second columns of $G^{2}$ become the first and second columns of $\bar{G}$.

\section{Identification by sign restrictions}

Under full sign restrictions, all of the $\theta_{i j}$ parameters in the Givens rotation matrix $G$ are generated. We present a method to generate their values so that the Givens rotation matrix formed from them is essentially equivalent to the orthogonal matrix that was proposed by Rubio-Ramírez, Waggoner, and Zha (2010). This matrix, which is denoted by $Q$, is obtained from a QR decomposition of a matrix whose columns are randomly drawn form a standard normal density.

For our method, first consider the matrix $G^{1}$ which is $G\left(\theta_{12}\right) G\left(\theta_{13}\right) G\left(\theta_{14}\right)$. Generate a $(4 \times 1)$ vector $Z$ with each element $z_{i}$ randomly drawn from a $N(0,1)$ distribution and normalize each element by the norm of $Z$. The normalized vector is $W$, where $w_{i}=z_{i} / \sqrt{z_{1}^{2}+z_{2}^{2}+z_{3}^{2}+z_{4}^{2}}$ for $i=1,2,3,4$. It is important to note the elements in $W$ are uniformly distributed on the unit 4-sphere. ${ }^{1}$ Then equate each element in the first column of $G^{1}$ with the corresponding element in the vector $W$. By Result 1 and equation (10), we obtain:

$$
\begin{gathered}
c_{12} c_{13} c_{14}=w_{1} \\
s_{12} c_{13} c_{14}=w_{2} \\
s_{13} c_{14}=w_{3} \\
s_{14}=w_{4}
\end{gathered}
$$

Divide equation (14) by equation (13) to get:

$$
t_{12}=\frac{w_{2}}{w_{1}}
$$

where we use the result that $\tan \left(\theta_{i j}\right)=\sin \left(\theta_{i j}\right) / \cos \left(\theta_{i j}\right)$ i.e. in current notation $t_{i j}=s_{i j} / c_{i j}$. Equation (17) gives

$$
\hat{\theta}_{12}=\arctan \left(\frac{w_{2}}{w_{1}}\right)
$$

Similarly, divide equation (15) by equation (14) to obtain

$$
t_{13}=s_{12} \frac{w_{3}}{w_{2}}
$$

from which we get

$$
\hat{\theta}_{13}=\arctan \left(s_{12}\left(\hat{\theta}_{12}\right) \frac{w_{3}}{w_{2}}\right)
$$


Finally, divide equation (16) by equation (15) to get

$$
t_{14}=s_{13} \frac{w_{4}}{w_{3}}
$$

from which we have:

$$
\hat{\theta}_{14}=\arctan \left(s_{13}\left(\hat{\theta}_{13}\right) \frac{w_{4}}{w_{3}}\right)
$$

We then calculate $G^{1}$ as $G\left(\hat{\theta}_{12}\right) G\left(\hat{\theta}_{13}\right) G\left(\hat{\theta}_{14}\right)$.

Second, consider the matrix $G^{2}$ which is $G\left(\theta_{23}\right) G\left(\theta_{24}\right)$. Generate a $(3 \times 1)$ vector $\tilde{Z}$ with each element $\tilde{z}_{i}$ randomly drawn from a $N(0,1)$ distribution and normalize each element by the norm of $\tilde{Z}$. The normalized vector is $\tilde{W}$, where $\tilde{w}_{i}=\tilde{z}_{i} / \sqrt{\tilde{z}_{1}^{2}+\tilde{z}_{2}^{2}+\tilde{z}_{3}^{2}}$ for $i=1,2$, 3. Similarly, the elements in the vector $\tilde{W}$ are uniformly distributed on the unit 3-sphere. Then equate each (non-zero) element in the second column of $G^{2}$ with the corresponding element in the vector $\tilde{W}$. By Result 2 and equation (12) this gives:

$$
\begin{gathered}
c_{23} c_{24}=\tilde{w}_{1} \\
s_{23} c_{24}=\tilde{w}_{2} \\
s_{24}=\tilde{w}_{3}
\end{gathered}
$$

Solve as before to obtain:

$$
\begin{gathered}
\hat{\theta}_{23}=\arctan \left(\frac{\tilde{w}_{2}}{\tilde{w}_{1}}\right) \\
\hat{\theta}_{24}=\arctan \left(s_{23}\left(\hat{\theta}_{23}\right) \frac{\tilde{w}_{3}}{\tilde{w}_{2}}\right)
\end{gathered}
$$

which we use to calculate $G^{2}$ as $G\left(\hat{\theta}_{23}\right) G\left(\hat{\theta}_{24}\right)$.

Third, consider the matrix $G\left(\theta_{34}\right)$. Generate a $(2 \times 1)$ vector $\bar{Z}$ with each element $\bar{z}_{i}$ randomly drawn from a $N(0,1)$ distribution and normalize each element by the norm of $\bar{Z}$. The normalized vector is $\bar{W}$, where $\bar{w}_{i}=$ $\bar{z}_{i} / \sqrt{\bar{z}_{1}^{2}+\bar{z}_{2}^{2}}$ for $i=1,2$ which is uniformly distributed on the unit 2-sphere. Then equate each (non-zero) element in the third column of $G\left(\theta_{34}\right)$ with the corresponding element in the vector $\bar{W}$. By equation (8) this produces $c_{34}$ $=\bar{w}_{1}$ and $s_{34}=\bar{w}_{2}$ from which we obtain:

$$
\hat{\theta}_{34}=\arctan \left(\frac{\bar{w}_{2}}{\bar{w}_{1}}\right)
$$

We then calculate the Givens rotation matrix as $G=G^{1} G^{2} G\left(\hat{\theta}_{34}\right)$. Note that the Givens matrix is drawn uniformly because the elements of the vector from which the generated coefficients are found are uniformly distributed on the unit $N$-dimensional sphere.

The Givens rotation matrix formed in this manner will produce Results in sign restrictions very similar to those produced from the orthogonal matrix in a QR decomposition of $W$, where $W$ is a $(4 \times 4)$ matrix whose columns are randomly drawn from a $N\left(0, I_{4}\right)$. The decomposition is $W=Q R$, where $Q$ is an orthogonal matrix and $R$ is upper triangular. To demonstrate the assertion, we calculated one hundred thousand of the $G$ and $Q$ matrices and from these we found the empirical distribution for each of the elements in the respective matrix. Figure 1 shows that the empirical distribution of each element in $G$ is practically indistinguishable from the empirical distribution of the corresponding element in $Q$, meaning that in practice both rotation matrices will produce very similar Results in sign restrictions. In Peersman (2005), values for the $\theta_{i j}$ parameters were obtained by drawing each of them from a uniform distribution over the interval $[0, \pi]$. In this case, the empirical distribution of several of the elements in the $G$ matrix (not shown) depart noticeably from the empirical distribution of the corresponding elements in the $Q$ matrix. The Results for the Givens matrix formed in this way are likely to be somewhat different to the Results for the Givens matrix formed in the manner above and for the rotation matrix obtained from the QR decomposition. 

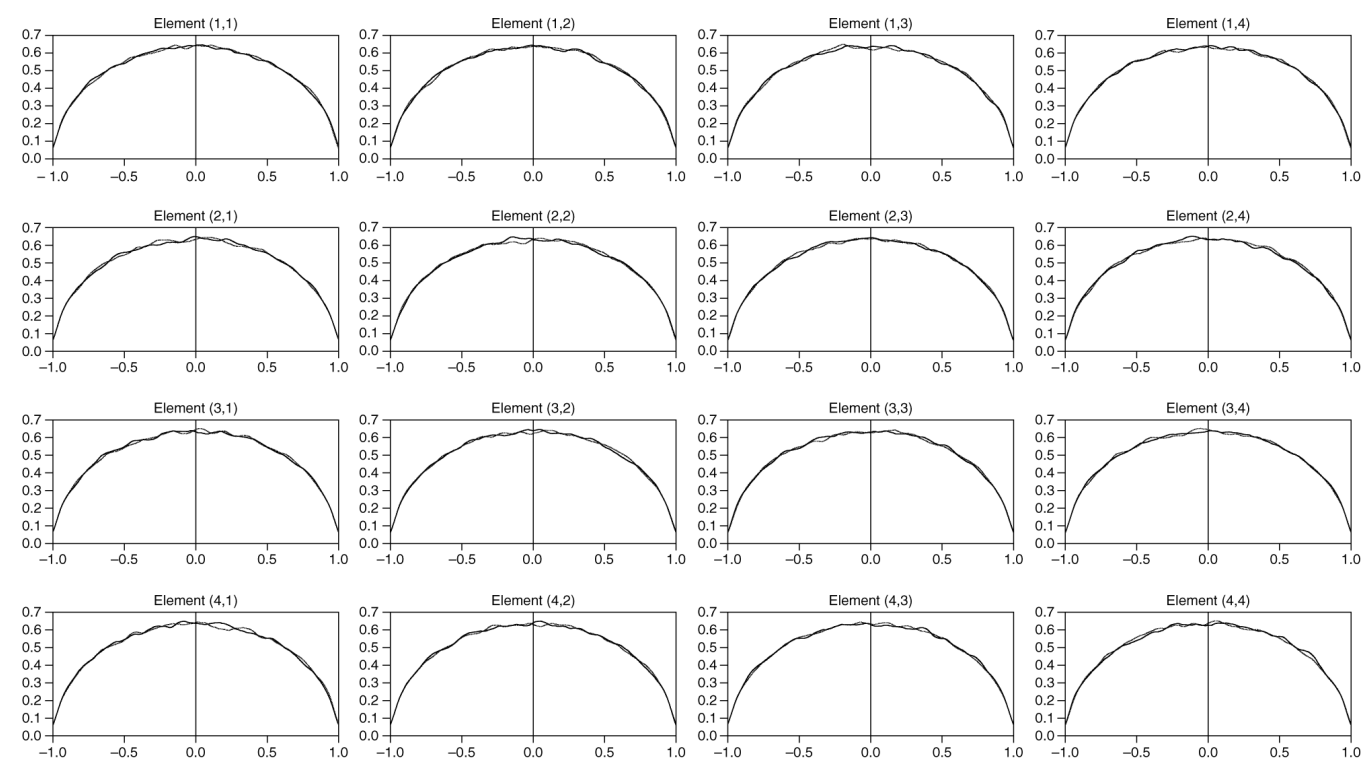

Figure 1: Empirical distribution of the elements in the Givens and $Q$ matrices, where $Q$ is from a QR decomposition. The empirical distribution for each element from the Givens rotation is shown with the thick line, while that from the $Q$ rotation in the QR decomposition is shown with the dotted line. Element $(i, j)$ refers to the element in the ith row and $j$ th column of a matrix.

The sign restrictions on the impulse responses utilised by Peersman (2005) are shown in Table 1. They separate the shocks as either a monetary policy (MP) shock, an aggregate demand (AD) shock, an aggregate supply (AS) shock or an oil price (OP) shock. In the table, the designation " $>0$ " denotes a positive response so that the variable rises in response to the shock while " $<0$ " denotes a negative response so that the variable falls in response to the shock. ${ }^{2}$ The designation "?" denotes an unrestricted response.

Table 1: Sign restrictions.

\begin{tabular}{|c|c|c|c|c|}
\hline Shock\variable & Oil price & Output & Consumer prices & Interest rate \\
\hline $\mathrm{OP}$ & $>0$ & $<0$ & $>0$ & $>0$ \\
\hline AS & $?$ & $>0$ & $<0$ & $<0$ \\
\hline $\mathrm{AD}$ & $>0$ & $>0$ & $>0$ & $>0$ \\
\hline MP & $<0$ & $<0$ & $<0$ & $>0$ \\
\hline
\end{tabular}

The designation " $>0$ " denotes a positive response, " $<0$ " a negative response and "?" an unrestricted response.

Peersman does not sign restrict the response of oil prices to an AS shock. In order to separate the OP shock from the AS shock, he treats the shock which has the largest contemporaneous effect on the oil price as the OP shock. This is a size restriction. The sign restrictions are standard. For example, they rule out "output" and "price" puzzles so that in response to an MP shock which raises the interest rate, oil prices, output, and consumer prices fall. The number of quarters over which the sign restrictions are applied to the impulse responses is four quarters for output and consumer prices and one quarter for the oil price and the interest rate.

Equations (13) to (28) present the method to obtain the Givens rotation matrix for the case when all of the shocks are separated by sign restrictions. Once the Givens rotation matrix is found, we obtain the impulse responses to the orthogonal shocks and either accept or reject them based on the sign restrictions in Table 1. The method is repeated to obtain another Givens rotation matrix which produces another set of orthogonal shocks to which impulse responses are obtained and judged by the sign restrictions. The procedure is repeated many times, usually until a predetermined number of impulse responses are accepted.

We now turn to the case of where both sign and parametric restrictions are utilised to identify the orthogonal shocks. 


\section{Identification by combining sign with parametric restrictions}

To demonstrate the approach, we combine four long-run exclusion restrictions with two sign restrictions to separate the orthogonal shocks in Peersman's four variable model. The long-run restrictions are motivated by long-run nominal neutrality. They are:

LR1: The MP shock has a zero long-run effect on output.

LR2: The MP shock affects the oil price and consumer prices proportionately by the same amount in the longrun.

LR3: The AD shock has a zero long-run effect on output.

LR4: The AD shock affects the oil price and consumer prices proportionately by the same amount in the longrun.

Peersman's (2005) parametric identification utilised two long-run restrictions, namely, LR1 and LR3, so that the MP and AD shocks were restricted to have a zero long-run effect on output. Because both of these shocks can potentially have a different long-run impact on oil and consumer prices, these shocks can affect relative prices in the long-run and have real effects, contrary to the hypothesis of long-run nominal neutrality. The remaining restrictions he utilised were that the AS, AD, and MP shocks had a zero contemporaneous effect on oil prices, and the MP shock had a zero contemporaneous effect on output. To impose nominal neutrality, Fisher, Huh, and Pagan (2016) replaced the oil price in Peersman's model by the relative price of oil, defined as the difference between the log oil price and the log of consumer prices. They identified the shocks parametrically by imposing both long-run and contemporaneous exclusion restrictions. The long-run restrictions were for the MP and AD shocks to have a zero long-run effect on both output and the relative price of oil, which imposes nominal neutrality. The remaining two restrictions were for the AD and AS shocks to have a zero contemporaneous effect on the relative oil price. By contrast, we impose the four long-run restrictions LR1 to LR4 for nominal neutrality directly in Peersman's original model and combine them with two sign restrictions to achieve full identification of the structural shocks.

In our example, there are two parametric restrictions on both the MP and AD shocks and none on the AS and OP shocks. As there are three free parameters in the first column of the matrix $G$, we can choose the first orthogonal shock to be either the MP or AD shock. We will treat the first orthogonal shock as the MP shock, because in the next section, we impose a further parametric restriction on it.

Recall equation (5), which shows the long-run response of the variables to the shocks, and denote the typical element of $D(1) A_{0}^{-1}$ as $b_{i j}$. The elements of $D(1) A_{0}^{-1}$ are obtained from estimation of the reduced-form VAR and the decomposition of $\Omega$. The restriction that the MP shock has a zero long-run impact on output (i.e. LR1), is obtained by multiplying the second row of $D(1) A_{0}^{-1}$ with the first column of $G$, (see equation (10)), and setting the Resulting expression to zero to obtain:

$$
b_{21} c_{12} c_{13} c_{14}+b_{22} s_{12} c_{13} c_{14}+b_{23} s_{13} c_{14}+b_{24} s_{14}=0
$$

Divide both sides of equation (29) by $c_{12} c_{13} c_{14}$ to obtain:

$$
b_{21}+b_{22} t_{12}+b_{23} \frac{t_{13}}{c_{12}}+b_{24} \frac{t_{14}}{c_{12} c_{13}}=0
$$

where $t_{i j}=s_{i j} / c_{i j}$.

Similarly, the restriction that the MP shock changes both oil prices and consumer prices proportionately by the same amount (say, by $k$ percent) in the long-run is obtained by multiplying the first and third rows of $D(1) A_{0}^{-1}$ with the first column of $G$ and setting the resulting expressions equal to $k$. Divide each expression through by $c_{12} c_{13} c_{14}$ to obtain, respectively,

$$
\begin{aligned}
& b_{11}+b_{12} t_{12}+b_{13} \frac{t_{13}}{c_{12}}+b_{14} \frac{t_{14}}{c_{12} c_{13}}=\frac{k}{c_{12} c_{13} c_{14}} \\
& b_{31}+b_{32} t_{12}+b_{33} \frac{t_{13}}{c_{12}}+b_{34} \frac{t_{14}}{c_{12} c_{13}}=\frac{k}{c_{12} c_{13} c_{14}}
\end{aligned}
$$


Equate equation (31) with equation (32) to get:

$$
\left(b_{11}-b_{31}\right)+\left(b_{12}-b_{32}\right) t_{12}+\left(b_{13}-b_{33}\right) \frac{t_{13}}{c_{12}}+\left(b_{14}-b_{34}\right) \frac{t_{14}}{c_{12} c_{13}}=0
$$

This is the expression for restriction LR2, which is that the MP shock affects oil and consumer prices by the same proportionate amount in the long-run.

We treat $\theta_{12}$ as the parameter which is generated and obtain its value $\hat{\theta}_{12}$ from equation (18) where now $w_{i}=z_{i} / \sqrt{z_{1}^{2}+z_{2}^{2}}$ for $i=1,2$ and $z_{i}$ is randomly drawn from a $N(0,1)$ distribution. Once $\hat{\theta}_{12}$ is obtained, $t_{12}$ and $c_{12}$ are also obtained because each is a function of $\theta_{12}$. Then equations (30) and (33) constitute a linear system of equations in the two unknowns $t_{13}$ and $t_{14} / c_{13}$ which can be solved as:

$$
\begin{aligned}
& t_{13}=c_{12}\left(\hat{\theta}_{12}\right) f^{1}\left(b_{i j}, t_{12}\left(\hat{\theta}_{12}\right)\right) \\
& \frac{t_{14}}{c_{13}}=c_{12}\left(\hat{\theta}_{12}\right) f^{2}\left(b_{i j}, t_{12}\left(\hat{\theta}_{12}\right)\right)
\end{aligned}
$$

where each solution is a function of the $b_{i j}$ parameters that appear in the system. ${ }^{3}$ From equation (34) we obtain the estimated value of $\theta_{13}$ as:

$$
\hat{\theta}_{13}=\arctan \left[c_{12}\left(\hat{\theta}_{12}\right) f^{1}\left(b_{i j}, t_{12}\left(\hat{\theta}_{12}\right)\right)\right]
$$

from which we can find $c_{13}$ since it is a function of $\theta_{13}$. Then from equation (35) we obtain the estimated value of $\theta_{14}$ as:

$$
\hat{\theta}_{14}=\arctan \left[c_{13}\left(\hat{\theta}_{13}\right) c_{12}\left(\hat{\theta}_{12}\right) f^{2}\left(b_{i j}, t_{12}\left(\hat{\theta}_{12}\right)\right)\right]
$$

We then calculate $G^{1}$ as $G\left(\hat{\theta}_{12}\right) G\left(\hat{\theta}_{13}\right) G\left(\hat{\theta}_{14}\right)$. Note that the first column of $D(1) A_{0}^{-1} G^{1}$ shows the long-run responses of the variables to the MP shock because it is identical to the first column of $D(1) A_{0}^{-1} G$ by Result 1 . In other words, the first column of $D(1) A_{0}^{-1} G^{1}$ is not affected by post-multiplication by $\bar{G}$ so that the parametric restrictions on the MP shock are maintained in the expression:

$$
C(1)=D(1) A_{0}^{-1} G^{1} \bar{G}
$$

where $G=G^{1} \bar{G}$.

We treat the second orthogonal shock as the AD shock. Denote the typical element of $D(1) A_{0}^{-1} G^{1}$ by $b b_{i j}$, which are found using the estimated $G^{1}$. The restriction that the AD shock has a zero long-run effect on output (i.e. LR3) is obtaining by multiplying the second row of $D(1) A_{0}^{-1} G^{1}$ by the second column of $\bar{G}$, and setting the resulting expression to zero. After dividing both sides by $c_{23} c_{24}$, we obtain:

$$
b b_{22}+b b_{23} t_{23}+b b_{24} \frac{t_{24}}{c_{23}}=0
$$

Similarly, and analogous to the earlier development, the restriction that the AD shock changes the oil price and consumer prices proportionately by the same amount in the long-run is:

$$
\left(b b_{12}-b b_{32}\right)+\left(b b_{13}-b b_{33}\right) t_{23}+\left(b b_{14}-b b_{34}\right) \frac{t_{24}}{c_{23}}=0
$$

Equations (39) and (40) constitute a linear system of equations in the two unknowns $t_{23}$ and $t_{24} / c_{23}$ which can be solved as:

$$
t_{23}=g^{1}\left(b b_{i j}\right)
$$




$$
\frac{t_{24}}{c_{23}}=g^{2}\left(b b_{i j}\right)
$$

where each solution depends on the parameters in the system. From equation (41), the estimated value of $\theta_{23}$ is:

$$
\hat{\theta}_{23}=\arctan \left[g^{1}\left(b b_{i j}\right)\right]
$$

and from equation (42), the estimated value of $\theta_{24}$ is:

$$
\hat{\theta}_{24}=\arctan \left[c_{23}\left(\hat{\theta}_{23}\right) g^{2}\left(b b_{i j}\right)\right]
$$

We then calculate $G^{2}$ as $G\left(\hat{\theta}_{23}\right) G\left(\hat{\theta}_{24}\right)$ and write the long-run impact matrix of the shocks as:

$$
C(1)=D(1) A_{0}^{-1} G^{1} G^{2} G\left(\theta_{34}\right)
$$

Note that the first and second columns of $D(1) A_{0}^{-1} G^{1} G^{2}$ show the long-run responses of the variables to the MP and AD shocks, respectively, because they are identical to the first and second columns of $D(1) A_{0}^{-1} G^{1} \bar{G}$ by Result 2. In other words, the first and second columns of $D(1) A_{0}^{-1} G^{1} G^{2}$ are not affected by post-multiplication by $G\left(\theta_{34}\right)$ so that the parametric restrictions on the MP and AD shocks are maintained. The parameter $\theta_{34}$ is generated using the method that led to equation (28) which gives its estimate. We then calculate $G$ as $G^{1} G^{2} G\left(\hat{\theta}_{34}\right)$ and obtain the impulse responses to the shocks from equation (4).

The parametric restrictions separate the MP and AD shocks from the AS and OP shocks. The former shocks have restricted long-run impacts on the variables whereas the latter do not. The sign restrictions on the responses of the oil price, consumer prices and the interest rate, shown in Table 1, separate the MP and AD shocks from each other. This separation does not require sign restricting the response of output to either of these shocks because both shocks have a zero long-run effect on output. The sign and size restrictions, shown in the table, separate the OP and AS shocks from each other.

We close this section with the following observations. First, we can impose at most three restrictions on the first shock, two on the second shock and one on the third shock. This corresponds to the number of free parameters in the first column of $G$, the number in the second column of $\bar{G}$, and the number in third column of $G\left(\theta_{34}\right)$. In the application here, two restrictions are imposed on the first shock and two on the second shock, leaving two of the $\theta_{i j}$ parameters to be generated. This accounting is analogous to the order condition which says that six restrictions are required for full parametric identification of a four-variable SVAR. ${ }^{4}$ This implies, for example, that with one more parametric restriction, one less $\theta_{i j}$ parameter is generated. Second, the two generated coefficients, $\theta_{12}$ and $\theta_{34}$, are drawn uniformly as the method which generates them utilises a vector whose elements are uniformly distributed on the unit 2-sphere.

We now turn to an extension of the application where additionally an exclusion restriction on the contemporaneous impact of a shock is imposed.

\section{An extension}

In the model of the previous section, there were four long-run parametric restrictions. We now consider the model under a fifth parametric restriction. This is a short-run exclusion restriction and it was also utilised by Peersman in his full parametric identification of the shocks. The restriction is:

SR1: The MP shock has a zero contemporaneous effect on output.

There are now three parametric restrictions on the MP shock. In this case, the MP shock can only be the first orthogonal shock because the only column of the matrix $G$ which has three parameters to be determined is the first one. The long-run restrictions on the MP shock are shown by equations (30) and (33). We now derive the equation for the short-run exclusion restriction.

Recall equation (6) which shows the contemporaneous response of the variables to the shocks and denote the typical element of $A_{0}^{-1}$ by $a_{i j}$, which are obtained from the decomposition of $\Omega$. The short-run restriction, SR1, is obtained by multiplying the second row of $A_{0}^{-1}$ with the first column of $G$, and setting the expression to zero. Divide through by $c_{12} c_{13} c_{14}$ to obtain: 


$$
a_{21}+a_{22} t_{12}+a_{23} \frac{t_{13}}{c_{12}}+a_{24} \frac{t_{14}}{c_{12} c_{13}}=0
$$

Equations (30), (33) and (46) constitute a linear system of equations in $t_{12}, t_{13} / c_{12}$ and $t_{14} / c_{12} c_{13}$ which can solved for:

$$
\begin{gathered}
t_{12}=f^{1}\left(a_{i j}, b_{i j}\right) \\
\frac{t_{13}}{c_{12}}=f^{2}\left(a_{i j}, b_{i j}\right) \\
\frac{t_{14}}{c_{12} c_{13}}=f^{3}\left(a_{i j}, b_{i j}\right)
\end{gathered}
$$

where each solution is a function of the $a_{i j}$ and $b_{i j}$ parameters that appear in the system. From equation (47), we obtain the estimated value of $\theta_{12}$ as:

$$
\hat{\theta}_{12}=\arctan \left[f^{1}\left(a_{i j}, b_{i j}\right)\right]
$$

from which we obtain $c_{12}\left(\hat{\theta}_{12}\right)$. Then from equation (48) we have:

$$
\hat{\theta}_{13}=\arctan \left[c_{12}\left(\hat{\theta}_{12}\right) f^{2}\left(a_{i j}, b_{i j}\right)\right]
$$

from which we obtain $c_{13}\left(\hat{\theta}_{13}\right)$. Equation (49) gives:

$$
\hat{\theta}_{14}=\arctan \left[c_{12}\left(\hat{\theta}_{12}\right) c_{13}\left(\hat{\theta}_{13}\right) f^{3}\left(a_{i j}, b_{i j}\right)\right]
$$

We then calculate $G^{1}$ as $G\left(\hat{\theta}_{12}\right) G\left(\hat{\theta}_{13}\right) G\left(\hat{\theta}_{14}\right)$. It is important to stress that this calculated $G^{1}$ matrix will not be the same as the calculated $G^{1}$ matrix in the previous model. With the addition of the restriction SR1, $\hat{\theta}_{12}$ is found from the solution of a system of equations and not as before from equation (18) which involved random draws i.e. it is solved for and not generated. Further, $\hat{\theta}_{13}$ and $\hat{\theta}_{14}$ here come from a different system of equations than before. The calculated $G^{1}$ matrix here and the associated Givens rotation matrix $G$ will be such that the three parametric restrictions on the MP shock are satisfied. The AD shock is the second orthogonal shock and the two long-run restrictions imposed on it are given by equations (39) and (40) from which we solve for $\hat{\theta}_{23}$ and $\hat{\theta}_{24}$ which are shown by equations (43) and (44), respectively. For these values, we calculate $G^{2}$ and then equation (28) is used to generate $\hat{\theta}_{34}$. With these estimates we calculate the matrix $G$ and obtain the impulse responses from equation (4). The parametric restrictions will be evident in the responses. With the addition of SR1, the $\mathrm{MP}$ and AD shocks are identified by the parametric restrictions alone. The sign restrictions are not required to separate them. It is still the case that the sign (and size) restrictions on the responses are required to separate the AS and OP shocks.

Finally, we can extend this model to a full parametric identification by adding a further restriction which, for the purposes of illustration, we take as:

SR2: The AS shock has a zero contemporaneous effect on the interest rate.

This restriction is similar to that used by Cushman and Zha (1997) and Kim and Roubini (2000). Here the parameter $\theta_{34}$ is no longer generated as in equation (28) but is found as the solution to an equation shown below. Here the third orthogonal shock is treated as the AS shock because one parametric restriction is placed on it and there is only one free parameter in the matrix $G\left(\theta_{34}\right)$. Denote the typical element of the matrix $A_{0}^{-1} G^{1} G^{2}$ as $a a a_{i j}$, which we have already calculated. The SR2 restriction is obtained by multiplying the fourth row of $A_{0}^{-1} G^{1} G^{2}$ with the third column of $G\left(\theta_{34}\right)$. Setting the resulting expression to zero and dividing both sides by $c_{34}$ we obtain:

$$
a a a_{43}+a a a_{44} t_{34}=0
$$


Solve this equation to get:

$$
\hat{\theta}_{34}=\arctan \left(\frac{-a a a_{43}}{a a a_{44}}\right)
$$

and calculate $G\left(\hat{\theta}_{34}\right)$ and $G$.

We note that the rank condition for identification of the structural shocks is satisfied here because the shocks for some order have 0-1-2-3 restrictions placed on them. Here the order is 3-2-1-0 i.e. three for the MP shock, two for the AD shock, one for the AS shock and zero for the OP shock. This is a result of Rubio-Ramírez, Waggoner, and Zha (2010) who derive the rank conditions for identification in SVARs under a wide range of situations. ${ }^{5}$

\section{Results}

The reduced-form VAR is estimated with Peersman's data for the United States (US). The data covers the period 1980:Q1 to 2002:Q2 and is obtained from the Journal of Applied Econometrics data archive. ${ }^{6}$ In line with Peersman, the VAR is specified with three lags and with a constant and a time trend in each equation, and we allow for estimation uncertainty in the parameters of the VAR. Following Peersman, the prior for the reduced-form VAR coefficients conditional on $\Omega$ is normal, and the prior on $\Omega$ is inverse-Wishart. Recall that the generated coefficients in the Givens rotation are drawn uniformly as the generation method utilises a vector whose elements are uniformly distributed on the unit N-sphere. In the terminology of Arias, Rubio-Ramírez, and Waggoner (2018), we are using a uniform-normal-inverse-Wishart distribution for the priors over the orthogonal reduced-form parameterization of the SVAR. This parameterization is characterised by the reduced-form coefficients, the covariance matrix of reduced-form errors $\Omega$, and the orthogonal rotation matrix, which here is our Givens matrix. The uniform part of the prior follows as the generated coefficients in our Givens matrix are drawn uniformly.

Having estimated the reduced-from VAR, we obtain an initial set of orthogonal shocks, rotate them using the Givens matrix, and calculate the impulse responses to the rotated shocks from equation (4). The impulse responses are calculated for the levels of the series and the set of responses are either accepted or rejected based on the applicable sign restrictions. Each time this procedure is repeated a different Givens rotation matrix is formed and a new set of responses are found which are judged for retention or not. The procedure is repeated until 1000 sets of responses are retained i.e. accepted.

The accepted responses are arranged into ascending order at each horizon and the median response is found. The medians are connected point-wise across horizons to form the median impulse response. We also find the 16th and 84th percentile response in this way. It is important to note that the median response (and, for that matter, a percentile response) at each horizon is likely to come from a different Givens rotation. Fry and Pagan (2011) refer to this as the multiple models problem. They propose a metric to find the single Givens rotation which minimises the distance between the accepted impulse responses and median responses for all of the shocks. The median-target responses are the responses produced by this Givens rotation matrix and they correspond to a single SVAR. Both the median and median-target responses are reported in the figures that follow together with the 16th and the 84th percentile responses with the region between them shaded.

Figure 2 shows the results under full sign identification. The median responses are very similar to those shown in Peersman (2005, figure 2a, 193). As discussed earlier, the draws for our Givens rotation matrix are from the standard normal whereas in Peersman they come from a uniform distribution, but that variation does not lead to results materially different from his. Figure 2 shows that there is a substantial impact and long-run response of oil prices to AS, AD and MP shocks whereas the impact and long-run response of consumer prices to these shocks is considerably smaller, in absolute terms. In response to the MP shock which raises the interest rate, the median response of oil prices shows a fall of 5 percent on impact and a fall of 8 percent in the long-run, whereas the median response of consumer prices shows a fall of 0.13 percent on impact and a fall of 0.5 percent in the long-run. A similar response pattern for oil and consumer prices is observed with respect to the AS and $\mathrm{AD}$ shocks. It is interesting to note that the median response of output to the AD and MP shocks is close to zero at long horizons, which is consistent with monetary neutrality. However, the AD and MP shocks affect oil prices proportionately much more than consumer prices at long horizons, thereby altering relative prices, which is not consistent with monetary neutrality. Specifically, the median response of oil and consumer prices to the AD shock show a rise of 5 and 0.1 percent, respectively, at long horizons while to the MP shock, they show a fall of 8 and 0.5 percent, respectively. Finally, we note that the acceptance rate (the number of accepted sets of responses divided by the number of draws of the Givens rotation matrix that were required to obtain them) was 0.0316 percent. 

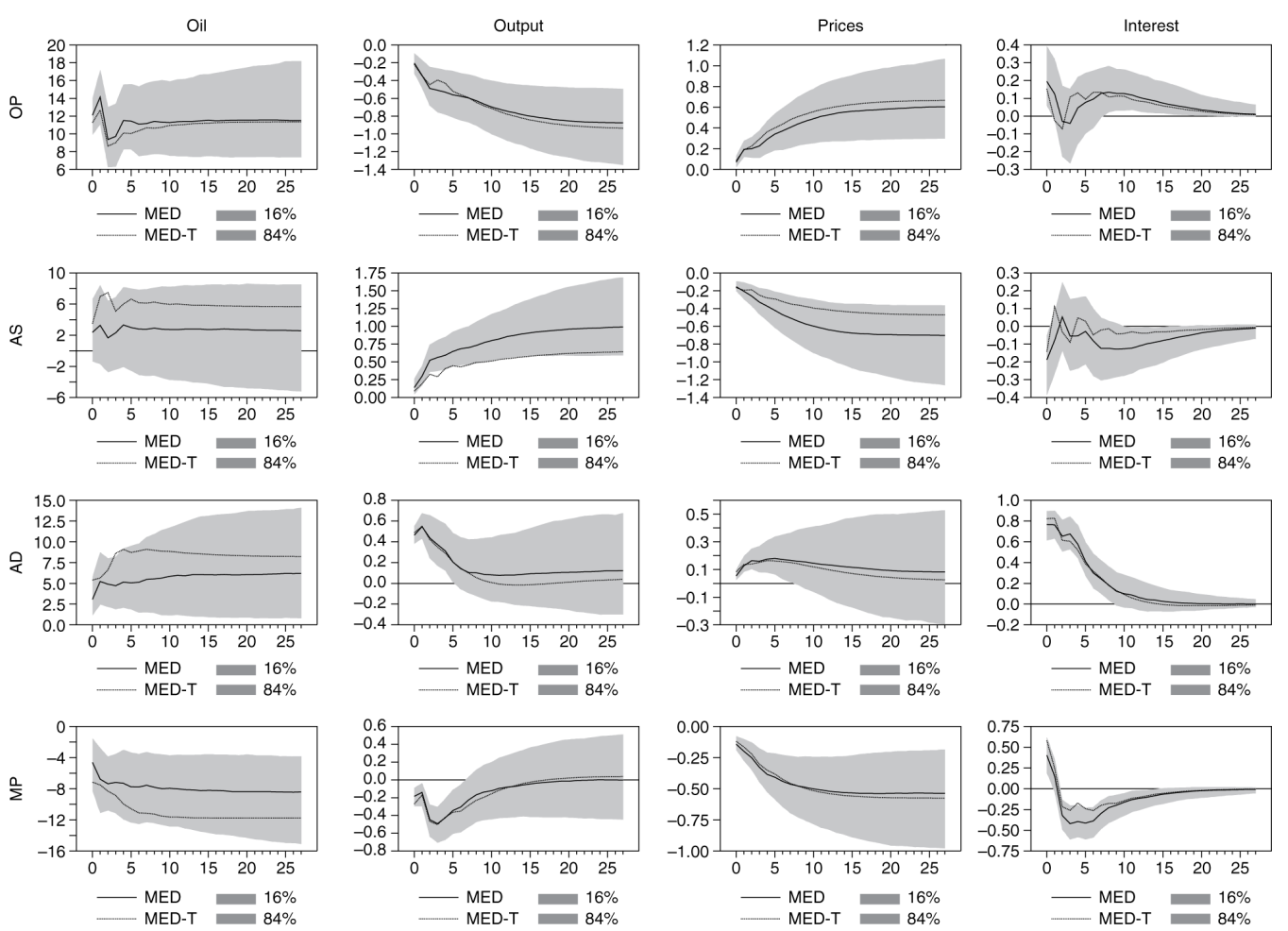

Figure 2: Full sign identification of Peersman's model.

Acceptance rate $=0.0316 \%$.

Figure 3 shows the results when sign restrictions are combined with the four long-run neutrality restrictions of Section 5. All of the output responses to the AD and MP shocks converge to zero in the long run as a consequence of the two restrictions LR1 and LR3. The oil and consumer price responses change by the same proportionate amount in the long-run in response to each of these shocks by restrictions LR2 and LR4. The median responses of oil and consumer prices to the AD shock show that they rise by about 0.07 percent in the long-run. The 16th percentile response shows a small decline while the 84th percentile shows a rise of about 0.4 percent in the long-run. With respect to the MP shock, the median response shows a fall in oil and consumer prices of 0.4 percent in the long-run while the percentile responses show a fall of between 0.3 and 0.5 percent. The median response shows a fall in output of 0.2 percent at the contemporaneous horizon in response to the MP shock. However, the 84th percentile response shows that output rises by 0.1 percent on impact following the MP shock so several of the responses show an output puzzle. ${ }^{7}$ An output puzzle can emerge here because the response of output to the AD and MP shocks was not sign restricted as that was not needed to separate them (see the discussion below equation (45)). It is also the case here that there is a substantial impact response of oil prices to the AS, AD and MP shocks, and that the impact response of consumer prices to these shocks is, respectively, considerably smaller. We note that for this combined identification, the acceptance rate is marginally higher at 0.0903 percent. 

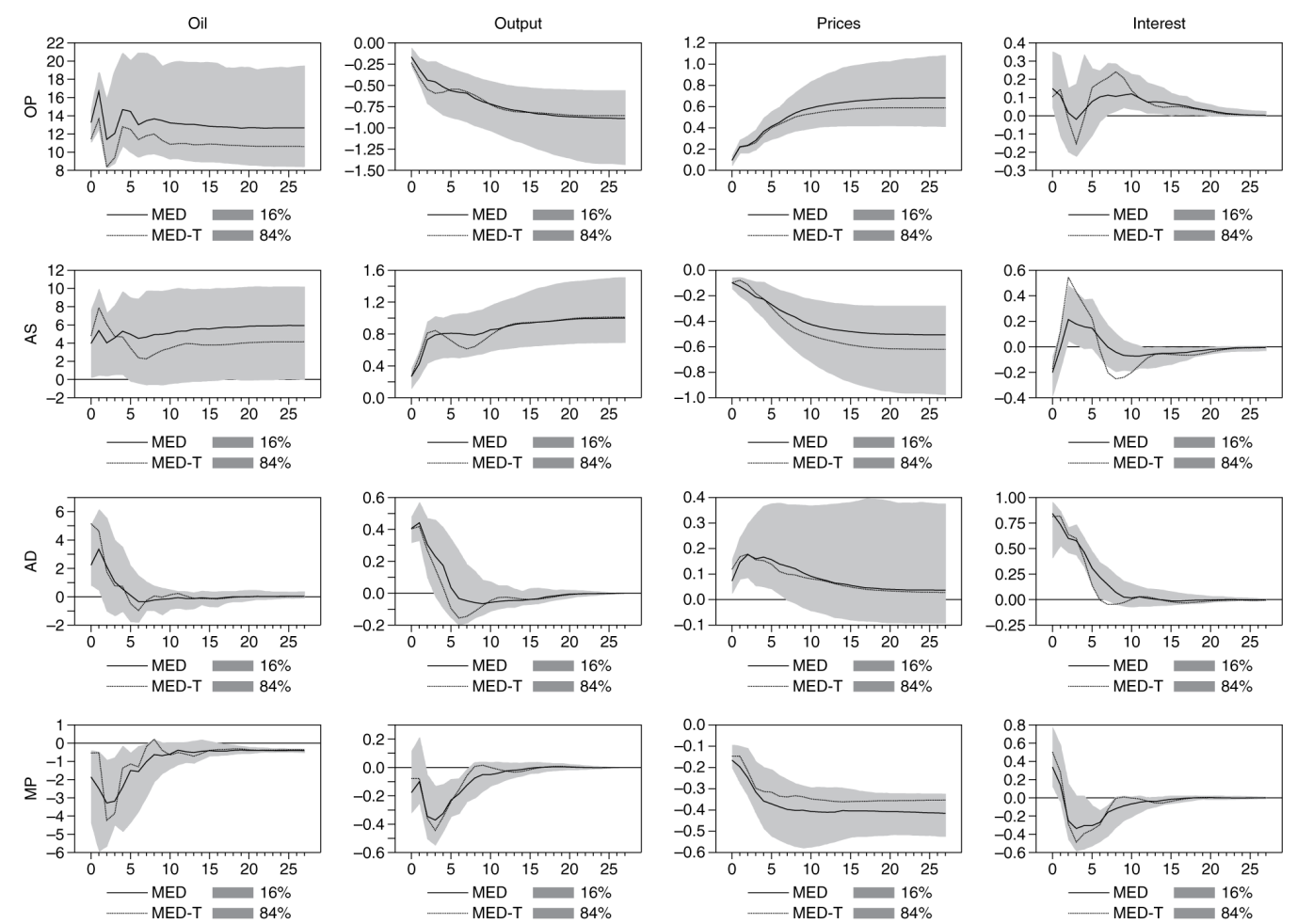

Figure 3: Sign and parametric identification of Peersman's model: Four long-run neutrality restrictions.

Acceptance rate $=0.0903 \%$.

Figure 4 shows the results for the extended identification of Section 6, which adds the contemporaneous zero restriction given by SR1 to the set of long-run restrictions. ${ }^{8}$ The long-run restrictions are evident in the responses as before. The long-run median responses of oil and consumer prices to the AD shock are somewhat higher at 0.25 percent and are about the same (-0.4 percent) to the MP shock. The output responses to both shocks all converge to zero at long horizons. The contemporaneous restriction is apparent in the response of output to the MP shock but one quarter after impact a slight output puzzle emerges. ${ }^{9}$ More troubling, however, is that a price puzzle emerges in the response of oil prices to the MP shock. The median impact response shows that oil prices rise by 1.5 percent and the 84th percentile response shows they rise by 4 percent, while the 16th percentile response shows a fall of 1 percent. While the median impact response of oil prices to the AD shock shows a small rise of 0.5 percent, several of the responses show a fall in view of the 16th percentile response which shows a fall of 2 percent. This is counter-intuitive. Under full parametric identification i.e. under LR1 to LR4, SR1 and SR2, the oil price and output puzzles persist, and there is a 2 percent fall in the median response of oil prices on impact to the AD shock, which is not a sensible finding. ${ }^{10}$ 

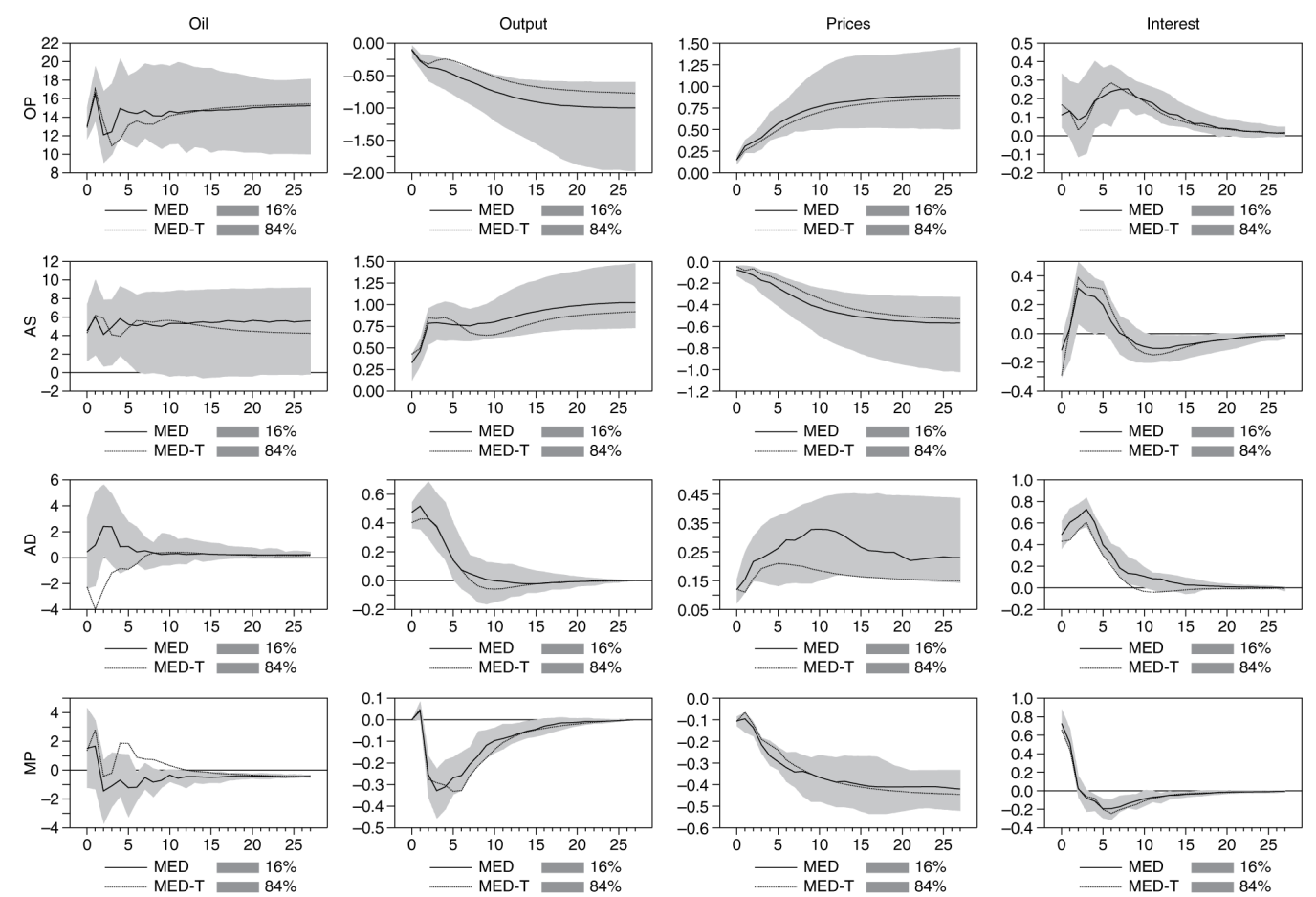

Figure 4: Sign and parametric identification of Peersman's model: Four long-run neutrality restrictions and the restriction that the MP shock has a zero contemporaneous effect on output.

Acceptance rate $=0.4688 \%$.

\section{Method of Arias, Rubio-Ramírez and Waggoner}

Arias, Rubio-Ramírez and Waggoner (2018) develop a method to combine sign and parametric restrictions. We demonstrate their method for the model of Section $5 .{ }^{11}$ In the ARW method, the initial set of orthogonal shocks are rotated by the matrix $Q$. The zero parametric restrictions are expressed as linear restrictions on the columns of $Q$ which are also restricted to be orthogonal to each other.

The impulse responses to the new set of orthogonal shocks is given by:

$$
C(L)=D(L) A_{0}^{-1} Q
$$

which is analogous to equation (4). Write $Q=\left[\begin{array}{llll}q_{1} & q_{2} & q_{3} & q_{4}\end{array}\right]$ where each $q_{i}$ is a $(4 \times 1)$ vector and, as before, let the typical element of $D(1) A_{0}^{-1}$ be $b_{i j}$. Suppose we treat the first shock as the MP shock. The ARW method finds the vector $q_{1}$ so that the two long-run restrictions LR1 and LR2 on the MP shock are satisfied i.e. it finds $q_{1}$ such that:

$$
R_{1} q_{1}=0
$$

where

$$
R_{1}=\left(\begin{array}{cccc}
b_{21} & b_{22} & b_{23} & b_{24} \\
b_{11}-b_{31} & b_{12}-b_{32} & b_{13}-b_{33} & b_{14}-b_{34}
\end{array}\right)
$$

and 0 is a $(2 \times 1)$ null vector. The elements in the rows of $R_{1}$ are the coefficients which correspond to LR1 and LR2, shown by equations (30) and (33), respectively. We then find the matrix $N_{1}$ whose columns form an orthonormal basis for the null space of $R_{1}$. The matrix $N_{1}$ can be found from a singular value decomposition of $R_{1}$, and in this case, it is a $(4 \times 2)$ matrix. ${ }^{12}$ Next draw a $(4 \times 1)$ vector $x_{1}$ from the standard normal distribution $N\left(0, I_{4}\right)$ and generate $q_{1}$, as $q_{1}=N_{1}\left(N_{1}^{\prime} x_{1} /\left\|N_{1}^{\prime} x_{1}\right\|\right)$, where $\|\bullet\|$ is the Euclidian norm.

The second shock is treated as the AD shock and the method finds the vector $q_{2}$ so that the two long-run restrictions LR3 and LR4 on the AD shock are satisfied and further that $q_{2}$ is orthogonal to $q_{1}$. These restrictions are: 


$$
R_{2} q_{2}=0
$$

where

$$
R_{2}=\left(\begin{array}{cccc}
b_{21} & b_{22} & b_{23} & b_{24} \\
b_{11}-b_{31} & b_{12}-b_{32} & b_{13}-b_{33} & b_{14}-b_{34} \\
q_{1}^{\prime} & &
\end{array}\right)
$$

We then find the matrix $N_{2}$ which forms an orthonormal basis for the null space of $R_{2}$, and this matrix will be $(4 \times 1)$. We draw $x_{2}$ from $N\left(0, I_{4}\right)$ and find $q_{2}$ analogously as above.

As there are no further parametric restrictions, the ARW method finds $q_{3}$ and $q_{4}$ so that they are orthogonal to each other and to $q_{1}$ and $q_{2}$. To find $q_{3}$, form $R_{3}=\left(\begin{array}{ll}q_{1} & q_{2}\end{array}\right)^{\prime}$ and find an orthonormal matrix $N_{3}$ of dimension $(4 \times 2)$ which forms a basis for the null space of $R_{3}$. Draw $x_{3}$ and find $q_{3}$ both as before. Finally, form $R_{4}=\left(\begin{array}{lll}q_{1} & q_{2} & q_{3}\end{array}\right)^{\prime}$ and find $N_{4}$ of dimension $(4 \times 1)$. Draw $x_{4}$ and find $q_{4}$ as above. The procedure produces an orthogonal $Q$ matrix which has the four long-run zero restrictions imposed on it, and for another draw of the four vectors $x_{1}, x_{2}, x_{3}$ and $x_{4}$, it produces another $Q$, also with the restrictions imposed on it. The way the $x$ vectors are drawn means that the $Q$ matrix, conditional on the parametric restrictions, is drawn uniformly. The ARW method uses a uniform-normal-inverse-Wishart distribution for the priors over the orthogonal reducedform parameterization of the SVAR. Here "orthogonal" refers to the matrix $Q$ and "uniform" to the fact that it is uniformly distributed, conditional on the parametric restrictions. ARW also refer to the structural representation of a SVAR which is characterized by its structural coefficients.

The method we have described is Algorithm 2 in Arias, Rubio-Ramírez, and Waggoner (2018, 697-698) and our description closely follows that of Kilian and Lütkepohl (2017,477-478). This algorithm independently draws from the uniform-normal-inverse-Wishart prior over the reduced-form parameterization and ARW show that it produces independent draws from the posterior distribution over the structural parameterization, conditional on the zero parametric restrictions. ARW show that this posterior distribution is proportional to a normalinverse-Wishart density weighted by a volume element over the structural parameterization. This means that when there are zero restrictions, the uniform-normal-inverse-Wishart prior is not conjugate for the structural representation. ARW then extend the algorithm to independently draw from a family of conjugate posterior distributions over the structural parameterization, conditional on the zero and sign restrictions, and they call this family normal-generalized-normal. Algorithm 2 is extended with a step for importance sampling and it becomes ARW's Algorithm $3(2018,700)$. This takes the posterior density from Algorithm 2 as the proposal distribution, the normal-generalized-normal density as the desired distribution and calculates the importance weight for each draw i.e. for each structural model that satisfies the sign and zero restrictions. The draws are re-sampled with replacement with probability proportional to their importance weight. In this way, Algorithm 3 draws independently from the normal-generalized-normal posterior over the structural parameterization, conditional on the sign and zero restrictions.

Given that the main expense of Algorithm 3 is the time required to compute the importance weights for each successful draw, ARW say that it is permissible to use Algorithm 2, particularly, "if one was not wedded to using the normal-generalized-normal distribution over the structural parameterization for the prior and posterior." (Arias, Rubio-Ramírez, and Waggoner 2018, 702). However, they do point out that the posterior distribution over the structural parameterization, conditional on the zero restrictions, produced by Algorithm 2 is not invariant to a re-ordering of the shocks, and that this issue does not arise with Algorithm 3.

In this paper, we show the results utilising Algorithm 2 of ARW i.e. for the algorithm described above. We do not undertake importance sampling for three reasons. First, the uniform-normal-inverse-Wishart distribution for the priors over the orthogonal reduced-form parameterization is common in the literature. As a very recent example, Arias, Caldara, and Rubio-Ramírez (2019) estimate their SVAR for this parameterization so it seems that even these authors are not particularly wedded to the normal-generalized-normal posterior. Second, the issue with respect to the re-ordering of the shocks does not arise in our application because identical longrun zero parametric restrictions are placed on the MP and AD shocks so whether the first shock is the MP shock and the second the AD shock, or vice-versa, has no impact on the results. Third, the Givens method has a uniform-normal-inverse Wishart prior over the orthogonal reduced-form parameterization, and it is not intuitively obvious what the posterior density over the structural parameterization, conditional on the sign and zero restrictions will be with this method. Whether there is a role for importance sampling in the Givens method will depend on what this posterior density is, and specifically, whether it is in the family of normal-generalizednormal densities over the structural parameterization, conditional on the sign and zero restrictions. To find this posterior density will be a challenging task best left for future research. To reiterate, the results in this paper are produced from algorithms that do not involve importance sampling. 
Figure 5 shows the results from the ARW method for Peersman's model under the sign and parametric restrictions of Section 5. A comparison of this figure with Figure 3 shows that these results are very similar to those from the Givens rotation method. A slight difference is that the output puzzle is somewhat more noticeable under ARW as the median and median target responses show a slight output puzzle one quarter after impact of the MP shock. Nevertheless, in both figures, several of the impact responses of output to the MP shock show an output puzzle based on the percentile responses. The acceptance rate under ARW is 0.0767 percent, which is nearly as much as the acceptance rate under Givens (0.0903 percent).
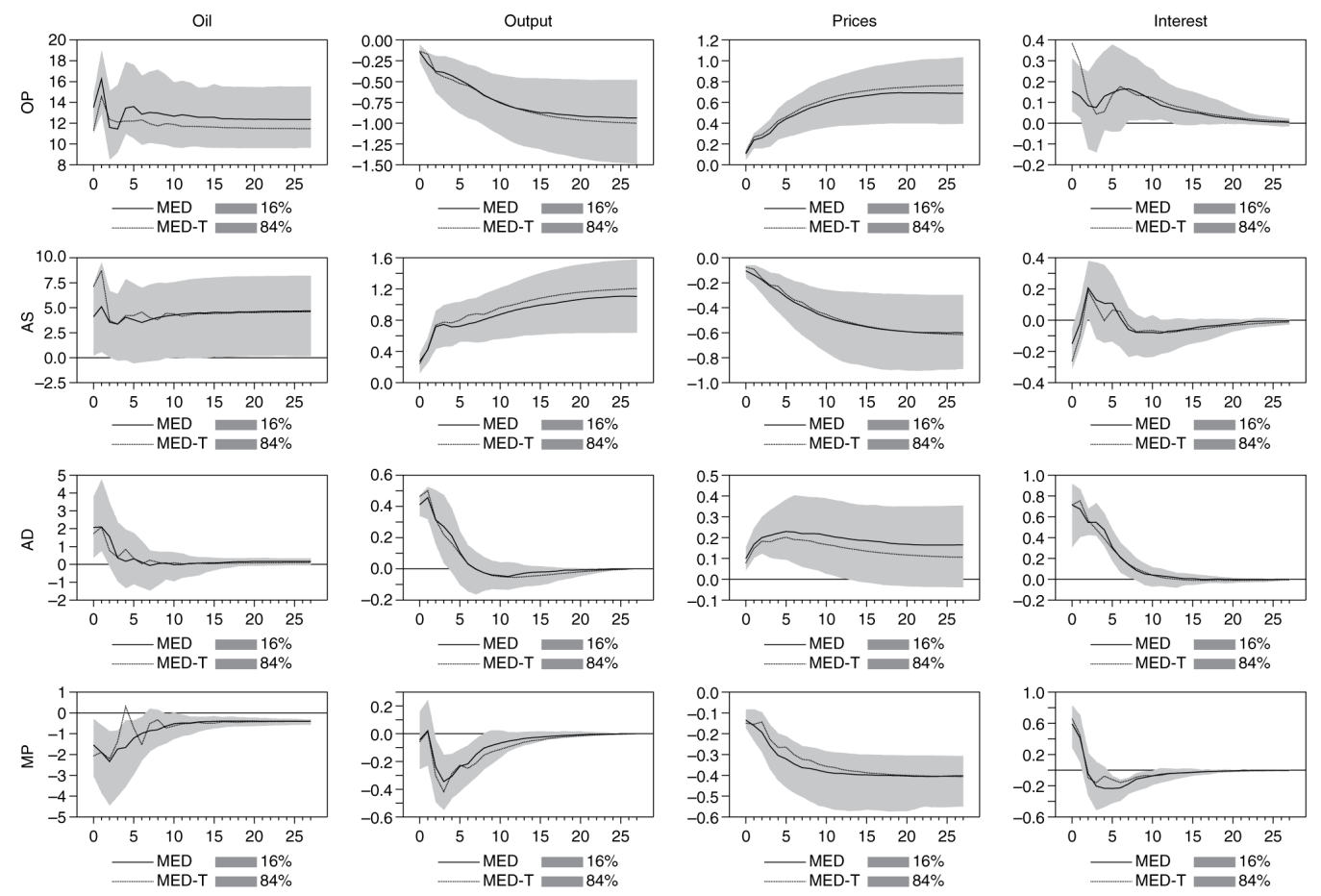

Figure 5: Sign and parametric identification of Peersman's model using Arias et al. method: Four long-run neutrality restrictions.

Acceptance rate $=0.0767 \%$.

The results in Figure 3 and Figure 5 are obtained using the software program RATS Version 9.1 on a personal computer with a $3.2 \mathrm{GHz}$ Intel Core i7-8700 processor and 16GB of RAM. For our Givens method (Figure 3) the computer running time was 8 minutes and 20 seconds whereas for the ARW method (Figure 5) the running time was 9 minutes and 28 seconds. ${ }^{13}$ While our Givens method is somewhat faster, the computational speed of both methods is close to each other. ${ }^{14}$ We attribute the similarity in computational speed to the fact that both methods draw the random vectors in the same way, namely, from a standard multi-variate normal distribution, as in the QR decomposition. As the number of variables in the SVAR increases, the relative computational efficiency may change, since there will be many more constituent Givens matrices in $G$ to calculate. However, there may not be much change if computational efficiency depends primarily on the generation method which is the same in both methods. ${ }^{15}$

\section{A comparison of the two methods}

In common with our Givens rotation method, the ARW method can impose at most three zero restrictions on the first shock in a four variable model, two on the second shock, one on the third shock and none on the fourth (Kilian and Lütkepohl, 2017,481). In the ARW method, the zero parametric restrictions are expressed as linear restrictions on the columns of the $Q$ matrix, which in turn, are restricted to be orthogonal. In our procedure, the parametric restrictions are imposed on the constituent Givens matrices, which form the $G$ matrix. As the Givens matrices are orthogonal, orthogonality restrictions are not needed.

Figure 6 shows the empirical distribution of each element in the matrix $Q$ along with the empirical distribution of the corresponding element in the matrix $G$ where both matrices are constructed under the four long-run zero parametric restrictions of Peersman. To form the empirical distributions, two hundred and fifty thousand of the $Q$ and $G$ matrices were calculated. Recall that the first and second columns of $Q$ and $G$ are constructed so that the two long-run parametric restrictions on the MP and AD shocks are satisfied on each draw, respectively. 
The empirical distributions of the corresponding elements in $Q$ in the first two columns of the figure are the same. This occurs because the parametric restrictions on the MP and AD shocks are the same and are imposed identically on the columns of the $Q$ matrix. This is not the case under our Givens method because the parametric restrictions are imposed on different constituent Givens matrices that form the matrix G. Importantly, the empirical distributions of the corresponding elements in $G$ and $Q$ in the first two columns of the figure are noticeably different in many instances. In particular, the empirical distribution of element $(2,1)$ in $G$ is noticeably peaked at zero whereas for $Q$ the distribution is bi-modal at the extremities. This may account for the somewhat more noticeable output puzzle under ARW. Note that the empirical distributions of each element in the third and fourth columns under both methods coincide because no parametric restrictions are placed on the third and fourth shocks.
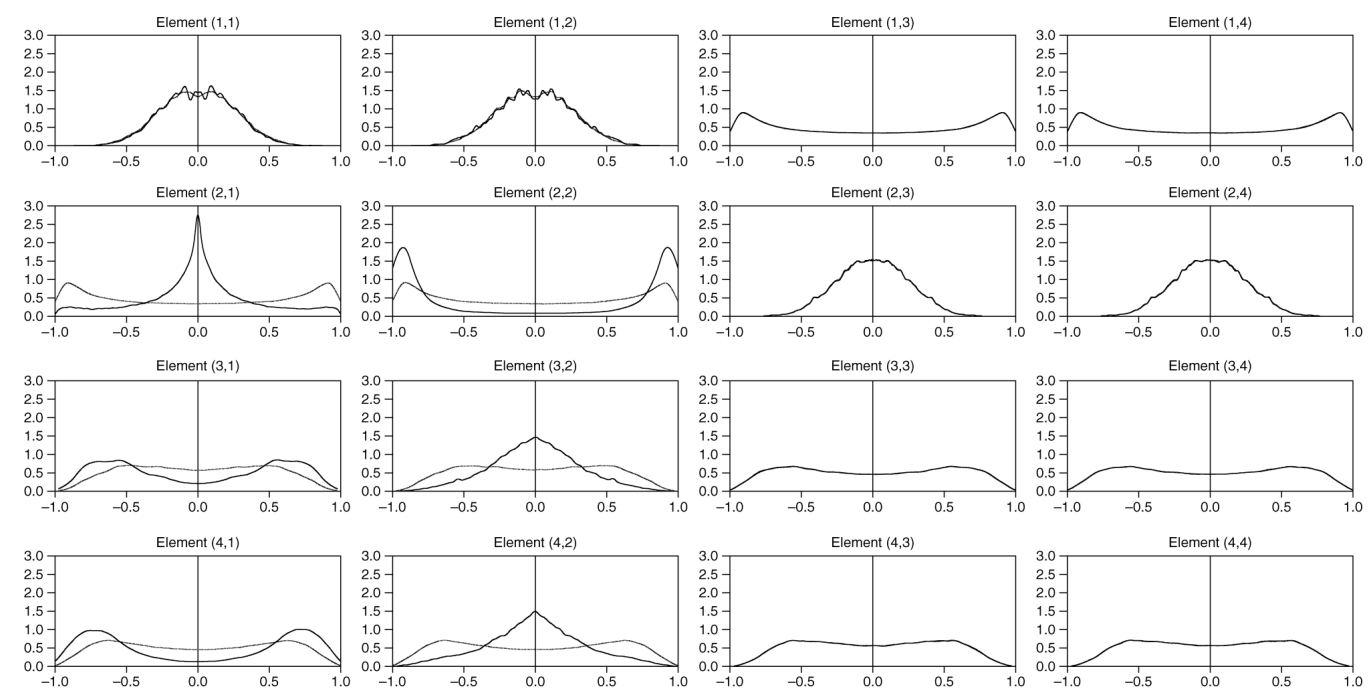

Figure 6: Empirical distribution of the elements in the Givens and $Q$ matrices when the four long-run neutrality restrictions are imposed.

The empirical distribution for each element from the Givens rotation is shown with the thick line, while that from the $Q$ rotation is shown with the dotted line. Element $(i, j)$ refers to the element in the $i$ th row and $j$ th column of a matrix.

The ARW method cannot be directly extended to imposing non-zero parametric restrictions on specific elements of the matrix of contemporaneous coefficients in the SVAR (Kilian and Lütkepohl, 2017, 481) or on the impulse responses. This is the case because the method involves finding a matrix whose columns are in the null space of the matrix of coefficients on the linear restrictions. The Givens method can accommodate nonzero parametric restrictions on the impulse responses and on the contemporaneous coefficients in the SVAR. As an example of the former, suppose the restriction that the MP shock has a zero long-run effect on output is replaced with the restriction that it has a non-zero long-run effect of $h$ percent. The right-hand side of equation (30), instead of being zero, is now $h / c_{12} c_{13} c_{14}$. This equation (30), along with equation (33), form a non-linear system of two equations in the two unknown angles, $\theta_{13}$ and $\theta_{14}$, (recall $\theta_{12}$ is generated) which can be solved for numerically. ${ }^{16}$ However, quantitative economic theory seldom delivers a precise non-zero restriction on an impulse response as, for example, that output will change by $h$ percent in the long-run following an MP shock. A more common situation is that there is extraneous information which provides a non-zero numerical value for a coefficient on a specific contemporaneous variable in a specific structural equation. This value can be interpreted as an elasticity if both variables are expressed in logs. For example, it could be known that the elasticity of the oil price with respect to output is $h$ so that the coefficient on contemporaneous output in the structural equation for the oil price is $h$. From $\varepsilon_{t}=G^{\prime} A_{0} e_{t}$ it follows that the matrix of contemporaneous coefficients in the SVAR is $G^{\prime} A_{0}$. Denote the typical element of $A_{0}$ as $a_{0}^{i j}$. This elasticity restriction is obtained by multiplying the first row of $G^{\prime}$ with the second column of $A_{0}$ and setting the resulting expression to $h$ to obtain:

$$
a_{0}^{12} c_{12} c_{13} c_{14}+a_{0}^{22} s_{12} c_{13} c_{14}+a_{0}^{32} s_{13} c_{14}+a_{0}^{42} s_{14}=h
$$

If this is the only restriction on the first column of $G$ i.e. first row of $G^{\prime}$, the angles $\theta_{12}$ and $\theta_{13}$ are generated and equation (60) is solved for the angle $\theta_{14}$. This restriction could also be used in combination with the restriction that the MP shock affects oil prices and consumer prices proportionality the same in the long-run. In this case, there are two restrictions on the first column of $G$, the angle $\theta_{12}$ is generated, and equations (33) and (60) are a non-linear system which is solved numerically for the angles $\theta_{13}$ and $\theta_{14}$. 


\section{Summary}

In this paper, we first describe a method for generating the parameters in the Givens rotation matrix for use in full sign restrictions. This method draws vectors randomly from a standard normal density as is done in a QR decomposition. This method is applied to Peersman's SVAR under full sign identification and the results were similar to his findings, even though the parameters of the Givens rotation matrix in his study were drawn from a uniform distribution. We then turn to our main objective which is to develop a method to combine parametric with sign restrictions utilising Givens rotation matrices. Under zero parametric restrictions, the method produces a linear system of equations which is solved recursively for the unknown parameters (or "angles") in the Givens matrices, conditional on the values of the parameters that are drawn. The constituent Givens matrices form the Givens rotations matrix such that the parametric restrictions on the responses are satisfied on each draw in sign restrictions. The method was applied to Peersman's SVAR under four long-run neutrality restrictions together with signs, and under these and a contemporaneous zero restriction. The former produced the most sensible responses. The recent method of ARW to combining zero restrictions with signs was applied to Peersman's SVAR under the long-run neutrality restrictions and produced similar results to Givens. Both methods are of similar computational efficiency with Givens slightly faster in our application. We show that the Givens approach can impose non-zero parametric restrictions on the responses and on the contemporaneous coefficients in the SVAR. In that respect, the Givens method is more general than the ARW method which can only impose zero parametric restrictions.

\section{Acknowledgement}

We would like to thank Adrian Pagan and two anonymous referees for helpful comments. The first author thanks Yonsei University for its generous hospitality.

\section{Funding}

The research by the first author was supported by a Macquarie University, Funder Id: http://dx.doi.org/10.13039/501100001230, OSP grant. The research by the second author is supported by the Ministry of Education of the Republic of Korea and the National Research Foundation of Korea (Funder Id: http:/ /dx.doi.org/10.13039/501100003725, NRF-2019S1A5A2A01038776).

\section{Notes}

1 This result is presented by Muller (1959) for the $N$ dimensional case

2 Peersman expressed the sign restrictions as weak inequalities, not as strict inequalities. In the case of weak inequalities, a common practice is to discard a draw for which there are zero responses, which would occur only rarely, as it may not be possible to separate all of the shocks. As an example, suppose in response to a shock there is a zero impact response of the oil price, output and consumer prices and a positive response of the interest rate, and suppose that also happens in response to another of the shocks. Under weak inequalities, these two shocks can be either an AD or an MP shock; they cannot be individually separated as one or the other, so strictly speaking Peersman's model is not fully sign identified. We thank a referee for pointing this out and for providing the example. To eliminate such cases, the sign restrictions are applied as strict inequalities here.

3 The system of linear equations can be solved recursively or by using Cramer's rule.

4 In a SVAR of $n$ variables, $n(n-1) / 2$ parametric restrictions are required to identify the structural shocks. This is the order condition. It is a necessary but not a sufficient condition for identification.

5 For example, if the shocks had 2-2-1-1 restrictions on them, the SVAR is not identified because the rank condition is violated. The order condition is satisfied but it is not sufficient for identification.

6 The URL for the Journal of Applied Econometrics data archive is: http://qed.econ.queensu.ca/jae/.

7 An output puzzle is said to occur when output rises on impact from a contractionary monetary policy shock. Similarly, a price puzzle is said to occur when prices rise on impact following a contractionary monetary policy shock.

8 The acceptance rate is 0.4688 percent, which is much higher than before, but this is due to the small role of sign (and size) restrictions in the identification. Here they are only needed to separate two of the shocks, namely, the OP and AS shocks.

9 This also shows up in Peersman's full parametric identification results which utilise two long-run restrictions (LR1 and LR3) and four contemporaneous restrictions, one of which is SR1.

10 The figure for this case is not shown but is available in the Supplementary materials.

11 Their method can easily handle the extended model of Section 6.

12 In RATS, the function \% $P E R P(R)$ returns an orthonormal matrix $N$ which forms a basis for the null space of the $R$ matrix i.e. $R N=0$.

13 The results were also generated on a more typical computer which had a $2.2 \mathrm{GHz}$ Intel Core i5-5200U processor and a 16GB of RAM. The Givens method took 14 minutes and 57 seconds while the ARW method took 17 minutes and 51 seconds. 
14 This is also the case under full sign identification. For Figure 2 the running time was 41 minutes and 31 seconds while for ARW under full signs (figure not shown but available in the Supplementary materials) it was 43 minutes and 8 seconds, both on the computer with the i7-8700 processor. The acceptance rate for ARW was 0.0313 percent, identical to that from Givens.

15 In early studies with sign restrictions, all possible Givens rotation matrices were computed over a finite-dimensional grid of values for each generated $\theta_{i j}$ parameter. In this case, we agree with Kilian and Lütkepohl (2017) and Fry and Pagan (2011) that the Givens method will likely become computationally burdensome as the number of variables in the SVAR increases but that is not what is done here.

16 The two unknown angles can be found using the utility "fsolve" in MATLAB or the instruction MAXIMIZE in RATS.

\section{References}

Arias, J. E., J. F. Rubio-Ramírez, and D. F. Waggoner. 2018. "Inference Based on Structural Vector Autoregressions Identified with Sign and Zero Restrictions: Theory and Applications." Econometrica 86 (2): 685-720.

Arias, J. E., D. Caldara, and J. F. Rubio-Ramírez. 2019. “The Systematic Component of Monetary Policy in SVARs: An Agnostic Identification Procedure." Journal of Monetary Economics 101: 1-13.

Baumeister, C., and L. Benati. 2013. “Unconventional Monetary Policy and the Great Recession: Estimating the Macroeconomic Effects of a Spread Compression at the Zero Lower Bound." International Journal of Central Banking 9 (2): 165-212.

Canova, F., and G. De Nicoló. 2002. "Monetary Disturbances Matter for Business Fluctuations in the G-7." Journal of Monetary Economics 49 (6): 1131-1159.

Cushman, D. O., and T. Zha. 1997. “Identifying Monetary Policy in a Small Open Economy under Flexible Exchange Rates." Journal of Monetary Economics 39: 433-448.

Faust, J. 1998. "The Robustness of Identified VAR Conclusions about Money." Carnegie-Rochester Conference on Public Policy 49: $207-244$.

Fisher, L. A., H.-S. Huh, and A. R. Pagan. 2016. “Econometric Methods for Modelling Systems with a Mixture of I(1) and I(0) Variables." Journal of Applied Econometrics 31 (5): 892-911.

Fry, R., and A. R. Pagan. 2011. "Sign Restrictions in Structural Vector Autoregressions: A Critical Review." Journal of Economic Literature 49 (4): 938-960.

Haberis, A., and A. Sokol. 2014. “A Procedure for Combining Zero and Sign Restrictions in a VAR-Identification Scheme.” CFM Discussion Paper Series, CFM-DP2014-10. Centre for Macroeconomics, London, UK.

Kilian, L., and H. Lütkepohl. 2017. Structural Vector Autoregressive Analysis. Cambridge, United Kingdom: Cambridge University Press.

Kim, S., and N. Roubini. 2000. "Exchange Rate Anomalies in the Industrial Countries: A Solution with a Structural VAR Approach." Journal of Monetary Economics 45 (3): 561-586.

Muller, M. 1959. "A Note on a Method for Cenerating Points Uniformly on N-Dimensional Spheres." Communications of the Association for Computing Machinery 2: 19-20.

Peersman, G. 2005. "What Caused the Early Millennium Slowdown? Evidence based on Vector Autoregressions." Journal of Applied Econometrics 20 (2): 185-207.

Rubio-Ramírez, J. F., D. F. Waggoner, and T. Zha. 2010. “Structural Vector-Autoregressions: Theory of Identification and Algorithms for Inference." Review of Economic Studies 77 (2): 665-696.

Uhlig, H. 2005. "What are the Effects of Monetary Policy on Output? Results from an Agnostic Identification Procedure." Journal of Monetary Economics 52 (2): 381-419.

Supplementary Material: The online version of this article offers supplementary material (DOI: https://doi.org/10.1515/snde-2018-0104). 